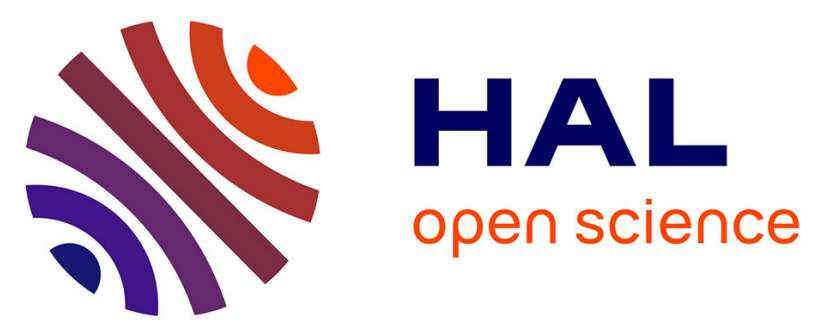

\title{
Quantitative analysis of bone microvasculature in mouse model using the monogenic signal phase asymmetry and marker-controlled watershed
}

\author{
Hao Xu, Max Langer, Françoise Peyrin
}

\section{- To cite this version:}

Hao Xu, Max Langer, Françoise Peyrin. Quantitative analysis of bone microvasculature in mouse model using the monogenic signal phase asymmetry and marker-controlled watershed. Physics in Medicine and Biology, 2021, 66 (12), pp.125005. 10.1088/1361-6560/ac047d . hal-03408070

\section{HAL Id: hal-03408070 \\ https://hal.science/hal-03408070}

Submitted on 28 Oct 2021

HAL is a multi-disciplinary open access archive for the deposit and dissemination of scientific research documents, whether they are published or not. The documents may come from teaching and research institutions in France or abroad, or from public or private research centers.
L'archive ouverte pluridisciplinaire HAL, est destinée au dépôt et à la diffusion de documents scientifiques de niveau recherche, publiés ou non, émanant des établissements d'enseignement et de recherche français ou étrangers, des laboratoires publics ou privés. 


\title{
Quantitative analysis of bone microvasculature in mouse model using the monogenic signal phase asymmetry and marker-controlled watershed
}

\author{
Hao $\mathrm{Xu}^{1}$, Max Langer ${ }^{1}$, Françoise Peyrin ${ }^{1,2}$ \\ ${ }^{1}$ Creatis, Université de Lyon, CNRS UMR5220, Inserm U1206, Université Lyon 1, INSA-Lyon, Villeurbanne, France. \\ ${ }^{2}$ European Synchrotron Radiation Facility (ESRF), Grenoble, France. \\ E-mail: Hao.Xu@creatis.insa-lyon.fr \\ Max.Langer@creatis.insa-lyon.fr \\ Francoise.Peyrin@creatis.insa-lyon.fr
}

\begin{abstract}
The three-dimensional (3D) imaging and quantitative analysis of bone microvasculature are important to describe angiogenesis involvement in bone metastatic processes. Here, we propose an algorithm based on marker controlled watershed for the 3D segmentation of vessels and bone in mouse bone imaged with a contrast agent using synchrotron radiation micro-computed tomography $(\mathrm{SR}-\mu \mathrm{CT})$. Markers were generated using hysteresis thresholding and morphological filters, and the control surface was constructed using the monogenic signal phase asymmetry. The accuracy and robustness of the proposed method were evaluated on a series of synthetic volumes generated to mimic the vessel, bone and background structures. Different contrast between different structures, as well as different noise levels were considered. A series of multi-class synthetic volumes were segmented using the proposed method, and the overall segmentation quality was evaluated using the Matthews correlation coefficient (MCC) by comparing to the ground truth. Additionally, we evaluated the segmentation of thin structures under various levels of Gaussian noise. The simulation study indicated that the algorithm was performant in multi-class segmentation with different contrast, noise, and thickness. The algorithm was applied to images of bone from a mouse model of breast cancer bone metastasis acquired using SR- $\mu \mathrm{CT}$. The segmentation quality was evaluated using the Dice coefficient and the MCC by comparing to manual segmentation. The proposed method performed better than hysteresis thresholding and marker-controlled watershed using the magnitude of the gradient as control surface. Several quantitative parameters on bone and vessels were extracted. The bone volume fraction (BV/TV) was significantly lower in the metastatic group compared to the healthy group. There was no significant difference on the vessel volume fraction (VV/TV) and the vessel thickness (VTh), possibly due to the limited sample size. This demonstrated the effectiveness of the algorithm for the study of bone and vessel microstructures in mouse model.
\end{abstract}

Keywords: micro-computed tomography, watershed, monogenic signal, bone, vessel, segmentation

\section{Introduction}

Breast cancer is the most frequently diagnosed cancer in women worldwide (Bray et al., 2018). In terms of mortality, there are approximately 508,000 deaths annually around the world (Tulotta et al., 2019). The major morbidity and mortality are caused by bone metastases, generally leading to osteolytic lesions. Around $70 \%$ of breast cancer patients develop to the advanced phase where tumors metastasize to bone (Akhtari et al., 2008). Once metastases locate in bone, the existing treatments are not able to cure the disease, and the median survival rate is about 24 to 65 months (Catarina et al., 2017; Lote et al., 1986; Nutter et al., 2014). 
Breast cancer bone metastases can not only bring about bone destructions but also facilitate the formation of undesirable vascularization (Nyangoga et al., 2011). To investigate and have a better understanding of the pathology, the three-dimensional (3D) imaging and analysis of bone and vasculatures are necessary.

3D X-ray microcomputed tomography $(\mu \mathrm{CT})$ is a tool of choice to image trabecular bone micro-architecture (Kuhn et al., 1990) as well as cortical bone and its Haversian network (Bousson et al., 2004; Cooper et al., 2003). In the study of assessing the effects of estrogen deficiency on bone, 3D X-ray $\mu \mathrm{CT}$ was used to image and analyze the rat bone structures (Sharma et al., 2018). Nevertheless, the protocol did not image the blood vessel to assess its alterations caused by the estrogen deficiency. In addition, 3D X-ray $\mu \mathrm{CT}$ can be used to visualize the vascular architecture with contrast agent (Moore et al., 2003; Zhang et al., 2005). However, these studies require to decalcify the bone. Therefore, $\mu \mathrm{CT}$ is only used to image and analyze either bone microstructure or vascular architecture separately, and these studies do not permit a complete analysis of the relationships between the bone and vasculature.

Synchrotron radiation $\mu \mathrm{CT}$ (SR- $\mu \mathrm{CT})$ has significant advantage of acquiring high spatial resolution images with a high signal-to-noise ratio, compared to the standard $\mu \mathrm{CT}$, due to the high photon flux of the synchrotron source (Salomé et al., 1999). SR- $\mu$ CT has been applied to the quantitative analysis of human trabecular bone up to the micrometer scale (Larrue et al., 2011; Peyrin et al., 1998). In addition, SR- $\mu$ CT coupled with the use of a contrast agent permitted to visualize simultaneously the $3 \mathrm{D}$ bone microstructures and vascular networks in mouse (Schneider et al., 2009) or rat (Prisby et al., 2011). Simultaneous visualizations of the calcified bone microstructure and blood vessels were also demonstrated using phase contrast synchrotron radiation computed tomography (SR-PCT) without any contrast agent in mice model (Núñez et al., 2017). However, in this study, the field of view was limited due to the small pixel size used, and vessels were segmented by hand due to the relatively weak phase contrast in the vessels limiting the applications of the protocol to large data sets.

The simultaneous imaging of bone and vessel is often followed by performing segmentation to separate bone and vessel compartments. The bone and vessel structures of mice, simultaneously imaged using SR- $\mu$ CT with a contrast agent, have been segmented automatically using the global thresholding (Schneider et al., 2009). Previously, in our group, 3D region growing has been proposed to segment rat bone vascularization (Langer et al., 2010; Prisby et al., 2011) in SR- $\mu$ CT images associated to a contrast agent. Hysteresis thresholding is a particular case of region growing with two thresholds. Specifically, voxels having greater intensities than the high threshold value are considered as the seeds of vessels, and regions grow from these seeds to include neighbors only if their intensities are between the high and low threshold values.

The aim of this work is to transfer the previous protocol to mice in order to make more models of pathologies available. However, this transition is not straightforward. Firstly, as opposed to in rat bone, vessels may appear to be in contact with the bone surface in mice, precluding the correct segmentation of bone and vessels using the previously proposed hysteresis thresholding based protocol, at $3.5 \mu \mathrm{m}$ resolution.

To address these challenges, we proposed to use the marker-controlled watershed algorithm (Beucher and Meyer, 1990) in conjunction with the monogenic signal phase asymmetry to segment and quantify the bone microstructure and vascular network in SR- $\mu$ CT images of mice. The proposed method was not only assessed on synthetic volumes, but also validated on experimental dataset by comparing to the manual segmentation. Segmentation using the proposed method was quantitatively compared to the hysteresis thresholding based method and gradient based markercontrolled watershed. To quantitatively analyze vessels and bone in the healthy and metastatic groups, several parameters were extracted to characterize bone microstructures and vasculatures.

\section{Methods}

\subsection{Image segmentation}

In this study, the aim of the segmentation method is to classify voxels into three classes: bone, vessels and background. The watershed algorithm can be used to separate vessels appearing to be touching the bone surface, without the need for post-segmentation merging of regions. The classical watershed transform is often used to segment an image into multiple objects by detecting the "catchment basins" and "ridge lines". In this method, the "flooding" begins from the regional minimums on the "control surface", which is treated as a topographic map and can be the magnitude of gradient. The ridge lines present in the image will then separate catchment basins when the different sources of water are merging. However, in most cases, there are many undesired regional minimums due to noise or natural variations, 
which lead to over-segmentation (Bhabatosh, 2011), requiring a posteriori merging of the segments. One way to avoid this problem is the marker-controlled watershed method, which initializes the watershed from the already identified markers (Beucher and Meyer, 1990). The method saves running time by reducing the number of iterations and prevents results from over-segmentation by simplifying the merging of the regions.

The proposed protocol was based on the marker-controlled watershed algorithm. In this work, we generated the markers of the different classes by using hysteresis thresholding and morphological filters, and constructed the control surface from the calculation of the monogenic signal phase asymmetry of the original image.

\subsubsection{Markers generation}

An important step of the marker-controlled watershed algorithm is to generate the marker image. To achieve coverage of all connected components in each class and minimize the false positives, the markers can be created using some feature detection methods or by hand (Wang and Vallotton, 2010). In this study, the marker generation procedure is introduced by using an example of mouse bone in SR- $\mu \mathrm{CT}$ image, as shown in Fig. 1. The markers of the three classes are generated using the hysteresis thresholding and morphological filters. The background and vessel markers are given by the cyan and magenta arrows, respectively. The initial segmentation is obtained using the hysteresis thresholding with two thresholds. The selections of the low and high threshold of hysteresis thresholding depend on the gray level distributions in the SR- $\mu \mathrm{CT}$ image. In this study, hysteresis thresholds were determined manually according to the testing results. We applied the same parameters on all samples, since all images had a similar gray level distribution. Then, the initial segmentation is followed by one iteration of morphological thinning to reduce the false-positive rate. The procedure to obtain bone marker is given by the yellow arrows. The whole foreground structure, containing both bone and vessel, is first segmented using hysteresis thresholding. Then, the dilated vessel markers are subtracted to obtain the initial bone markers. Since the aim of morphological dilation was to remove "halo" structures caused by subtraction, a small size of 3 voxels $(10.5 \mu \mathrm{m})$ of ball structuring element was enough in this study. Lastly, the isolated small particles are removed using morphological opening, and minimizing false positives is achieved by using morphological thinning. The final marker image is generated by adding the background markers, vessel markers, and bone markers, in cyan, magenta, and yellow, respectively.
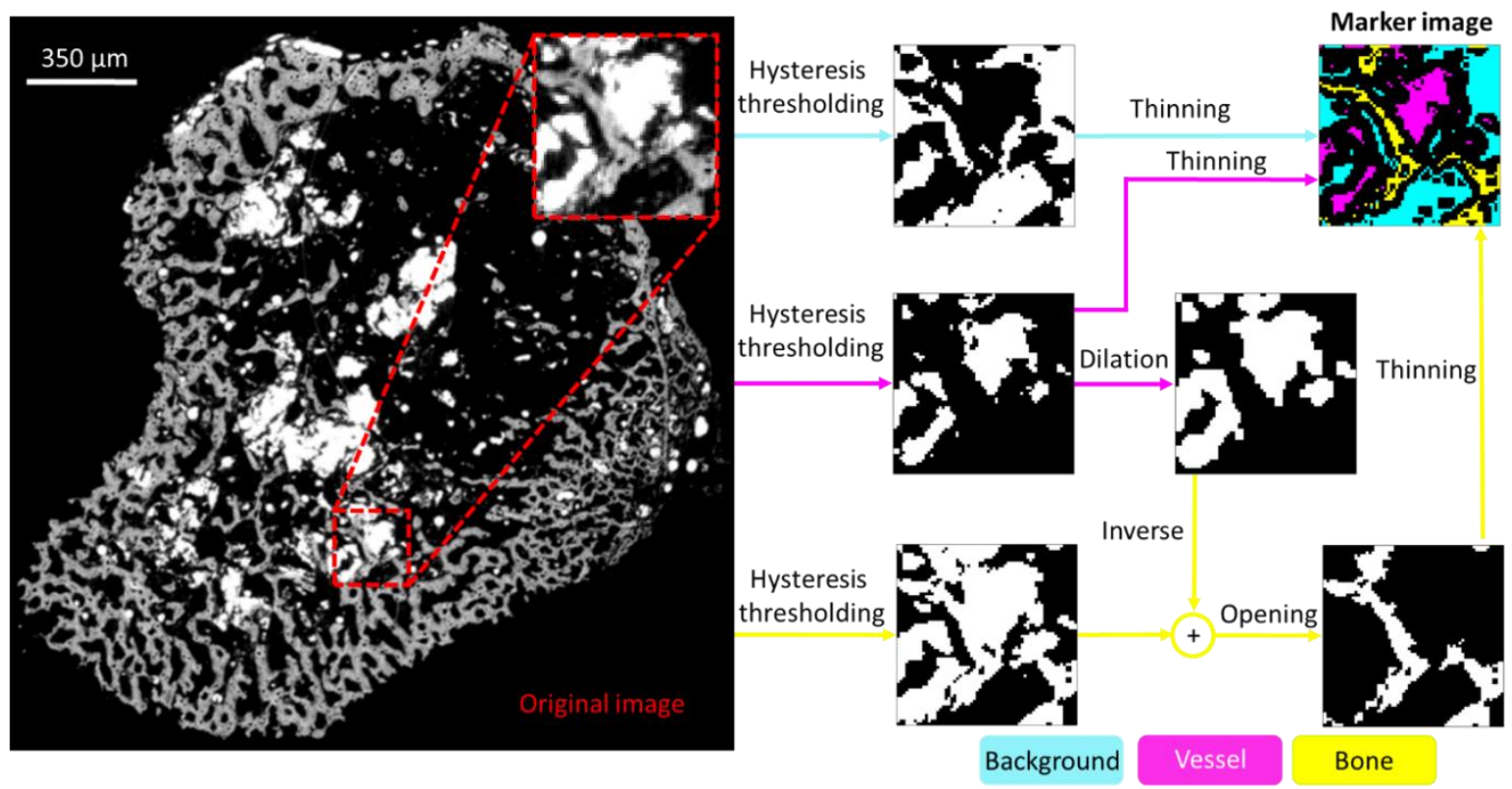

Fig. 1. The generation procedures of the marker images for the marker-controlled watershed.

\subsubsection{Control surface generation}

The other essential step of marker-controlled watershed algorithm is the generation of a control surface from the original image. Classically, the magnitude of the gradient is used as the control surface, which is related to intensity changes in the original image (Bhabatosh, 2011). However, in our case, the contrast at the interfaces between bone 
and vessels is relatively weak compared to the bone-background and the vessel-background interfaces, leading to weaker gradient magnitude at these edges. To alleviate this problem, we proposed the local phase asymmetry of the 3D monogenic signal as the control surface to improve the edge detection at the low contrast interfaces. The phase asymmetry measurement can be used to quantify whether the signal is locally edge like or line like. Since we aim to detect the interfaces between different structures, we use this property to select only the edge like structures for the control surface.

The 3D monogenic signal can be seen as the isotropic and multidimensional extension of the 1-D analytic signal (Felsberg and Sommer, 2001), and can be defined by the combination of the original signal and its three Riesz transform components (Chenouard and Unser, 2012):

$$
\begin{aligned}
& f_{m}(\boldsymbol{x})=\left(f(\boldsymbol{x}), R_{1} f(\boldsymbol{x}), R_{2} f(\boldsymbol{x}), R_{3} f(\boldsymbol{x})\right) \\
& \boldsymbol{R} f(\boldsymbol{x})=\left(R_{1} f(\boldsymbol{x}), R_{2} f(\boldsymbol{x}), R_{3} f(\boldsymbol{x})\right)
\end{aligned}
$$

where $f(\boldsymbol{x})$ denotes the original 3D image, and $\boldsymbol{x}=\left(x_{1}, x_{2}, x_{3}\right) \in \mathbb{R}^{3}$ is the spatial coordinate. $\boldsymbol{R}$ stands for the Riesz transform, and its operator component $R_{\alpha}: \mathbb{R}^{3} \mapsto \mathbb{R}$ is characterized by its frequency response:

$$
\mathcal{F}\left[R_{\alpha} f\right](\boldsymbol{\omega})=-j \frac{\omega_{\alpha}}{\|\boldsymbol{\omega}\|} \hat{f}(\boldsymbol{\omega}), \alpha=1, \ldots, 3
$$

where, $\mathcal{F}$ represents the Fourier transform operator, $j$ is the imaginary unit, $\boldsymbol{\omega}=\left(\omega_{1}, \omega_{2}, \omega_{3}\right) \in \mathbb{R}^{3}$ is the angular frequency variable conjugate to $\boldsymbol{x}$. $\alpha$ gives the 3D direction of the Riesz transformed components, corresponding to the directions of the basis vectors. In addition, structure features must be selected with an appropriate scale using a band-pass filter, for example a Log-Gabor filter with a shape parameter to govern the bandwidth of the passband, since structure in an image is generally scale dependent (Bridge, 2017). Thus, the 3D monogenic signal $f_{m g}(\boldsymbol{x})$ is constructed as:

$$
f_{m g}(\boldsymbol{x})=\left(f(\boldsymbol{x}) * g(\boldsymbol{x}), R_{1} f(\boldsymbol{x}) * g(\boldsymbol{x}), R_{2} f(\boldsymbol{x}) * g(\boldsymbol{x}), R_{3} f(\boldsymbol{x}) * g(\boldsymbol{x})\right)
$$

where $g(\boldsymbol{x})$ stands for Log-Gabor filter in spatial domain, and * represents the 3D convolution operation. The 3D monogenic signal can also be constructed as the combination of its even and odd components (Rajpoot et al., 2009):

$$
f_{m g}(\boldsymbol{x})=f_{m g e}(\boldsymbol{x})+j \cdot f_{m g o}(\boldsymbol{x})
$$

where the even component is

$$
f_{m g e}(\boldsymbol{x})=f(\boldsymbol{x}) * g(\boldsymbol{x})
$$

and the odd component is

$$
f_{m g o}(\boldsymbol{x})=\left(\sum_{\alpha=1}^{3}\left|R_{\alpha} f(\boldsymbol{x}) * g(\boldsymbol{x})\right|^{2}\right)^{1 / 2} .
$$

To detect edges in the original image, the multiscale phase asymmetry is measured by (Bridge, 2017):

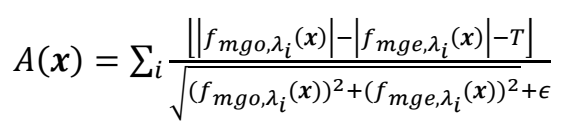

where [.] denotes an operator replacing negative values with zero, and $\left\{\lambda_{i}\right\}$ are a set of center-wavelengths $\left(\lambda_{i}=2 \pi / \omega_{i}\right)$ of the Log-Gabor filters to define the scales. $T$ is a threshold to suppress noise, $\epsilon$ is small number to avoid division by zero. In this study, we set multiscale values of $\lambda_{i}=6,7,8, T=0.18$ and $\epsilon=0.001$.

\subsection{Segmentation evaluation}

To evaluate segmentation quality, the Dice coefficient and Matthews correlation coefficient (MCC) were measured by comparing to manual segmentation or ground truth.

\subsubsection{Dice coefficient}

The Dice coefficient (also known as Dice similarity coefficient (DSC) and F1 score) is a statistic parameter to assess spatial overlapping between the segmentation and ground truth (Zou et al., 2004). Its expression is given by: 


$$
D S C=\frac{2|A \cap B|}{|A|+|B|}
$$

where $|A|$ and $|B|$ are the cardinalities of the two sets $\mathrm{A}$ and $\mathrm{B}$, representing respectively the segmentation and ground truth (Aaron et al., 2020).

\subsubsection{Matthews correlation coefficient}

The MCC (Matthews, 1975) is widely used as a measure of quality of classifications to indicate how well observations (ground truth) and predictions (segmentation) agree. MCC $=1$ indicates a perfect classification, MCC $=0$ indicates no better than random prediction, and MCC $=-1$ indicates a total misclassification (Matthews, 1975). In its original form, MCC applies to measure the quality of two-class classifications. However, a multi-class MCC has been proposed (Gorodkin, 2004):

$$
M C C=\frac{\sum_{k, l, m=1}^{N} C_{k k} C_{l m}-C_{k l} C_{m k}}{\sqrt{\sum_{k=1}^{N}\left(\sum_{l=1}^{N} C_{k l}\right)\left(\sum_{\begin{array}{c}
l^{\prime}=1 \\
k^{\prime} \neq k
\end{array}}^{N} C_{k^{\prime} l^{\prime}}\right)} \sqrt{\sum_{k=1}^{N}\left(\sum_{l=1}^{N} C_{l k}\right)\left(\begin{array}{c}
\sum_{l^{\prime}=1}^{N} C_{l^{\prime} k^{\prime}} \\
k^{\prime} \neq k
\end{array}\right)}}
$$

where $i j$-th entry $C_{i j}$ is the number of elements of observation class $i$ assigned to class $j$ (Jurman and Furlanello, 2010):

$$
C_{i j}=\mid\{\text { observation class }=i, \text { prediction class }=j\} \mid
$$

For instance, in a 3D image, $C_{k k}$ indicates how many voxels are correctly segmented in class $k$.

\subsection{Numerical simulations}

In order to examine the accuracy and robustness of the proposed method, as well as its generalization to other applications, four kinds of 3D synthetic models were created to simulate real datasets (Fig. 2). Each volume was an 8 bits 3D image with $256 \times 256 \times 150$ voxels. The size of synthetic phantom was determined by striking a balance between the size of real bone in SR- $\mu \mathrm{CT}$ images and computational cost. The gray levels of compartments in synthetic phantoms were set based on the real SR- $\mu \mathrm{CT}$ images. For instance, vessel and background were set at 255 and 0 in phantoms respectively, due to the contrasted vessel (bright) and background (dark) in the real SR- $\mu \mathrm{CT}$ images. As for the gray level of bone, it was set at different values between 0 and 255 to mimic the various contrast. The virtual phantoms were created based on geometrical cylinders and hourglass solids assigned 0,128 , and 255 greyscale values in their initial instances (Fig. 2). Additionally, in Model-1, Model-2, and Model-3, the diameters of the widest and narrowest hourglass solids were set to 34 and 1 voxels, respectively. In Model-4, the narrowest gap between the bright and gray structures were set to 1 voxel.

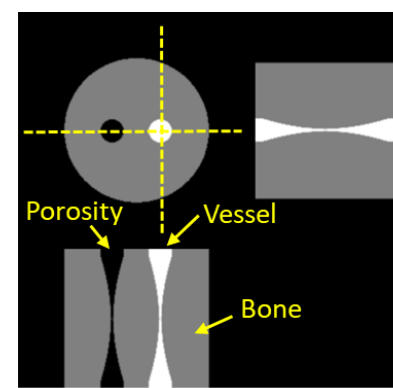

(a) Model-1

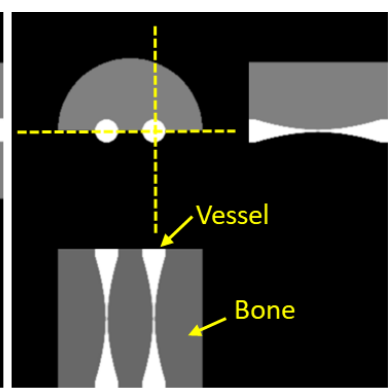

(b) Model-2

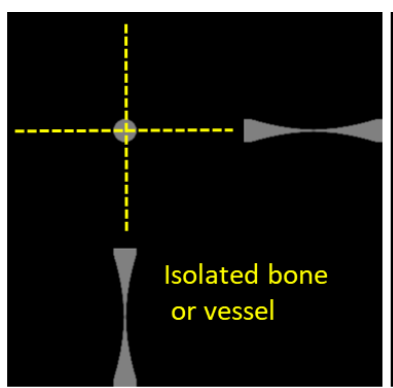

(c) Model-3

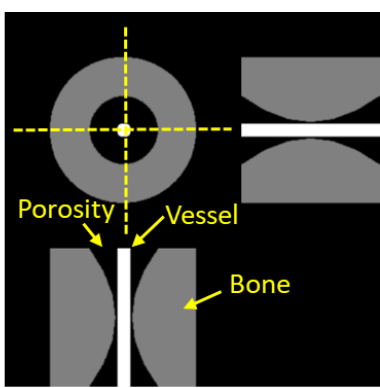

(d) Model-4

Fig. 2. Three orthogonal slices in the 3D synthetic models: (a) Model-1 represents vessel structure and bone porosity with various thickness, which are embedded in the bone. (b) Model-2 simulates that vessels are in contact with the bone surface and the background at the same time. (c) Model-3 represents an isolated structure (bone or vessel) in the real-world dataset. (d) Model-4 simulates a vessel passing through a canal (porosity) with varying diameter.

Specifically, Model-1 was designed to simulate 3D vessel structure (gray level 255) and bone porosity (background, gray level 0) with various thicknesses, surrounded by bone structure (gray level 128), as shown in Fig. 2 (a). Model- 
2 mimicked that vessels (gray level 255) were in contact with bone surface (gray level 128) and background (gray level 0) at the same time, as shown in Fig. 2 (b). Model-3 was designed to simulate an isolated structure in the realworld dataset, as shown in Fig. 2 (c). Model-4 described a situation that a vessel (gray level 255) was passing through a varying bone porosity (gray level 0), as shown in Fig. 2 (d). The bright cylinder in the center simulated vessel, and the surrounding structure was the synthetic bone (gray level 128).

To investigate the impact on the segmentation of different contrast levels between bone and vessels in the acquired images, a series of volumes were generated by varying the relative contrast of bone. Volumes were generated where the part representing bone was set to a value in the range 48 to 208, in increments of 10 gray levels (thus 17 volumes per model). For Model-3, an additional volume was generated where the structure was set to gray value 255 .

For the purpose of mimicking the partial volume effect, which is a reduction of gray level in small object and interface between two classes, the phantom images were downscaled and re-upscaled by a factor 4 with the bilinear resampling method. The synthetic phantoms without partial volume effects were used as reference images (ground truth).

In addition, to explore the impact of noise, different levels of Gaussian noise were added to each volume. The noise level was defined by the standard deviation of Gaussian noise. Noise was added with levels in a range of 10 to 70 with increments of 10, producing 63 instances of Model-1, Model-2, and Model-4, and 70 instances of Model-3.

\subsection{Application to experimental data}

\subsubsection{Sample preparation}

To validate the proposed algorithm on experimental data, we selected two groups of healthy and metastatic mice bone (10 samples per group) from a project investigating the effects of anti-angiogenic drugs (vatalanib: $100 \mathrm{mg} / \mathrm{kg}$ orally daily; bevacizumab: $5 \mathrm{mg} / \mathrm{kg}$ intraperitoneally twice a week) on metastasis formation. Specifically, 8 week-old female Balb/c nude mice were injected with luciferase-expressing human B02 breast cancer tumor cells (Fradet et al., 2011), then the preventive treatments were performed. In this model, animals usually develop bone metastases 18 days after the injection of tumor cell (Bachelier et al., 2014). Thus, the mice were sacrificed on 22 days after the injection. Next, all mice were injected with a contrast agent (barium sulfate) for the vascular imaging. Afterwards, mice tibiae were dissected, included in PMMA and kept for SR- $\mu$ CT imaging. The protocol (DR2015-18) was studied by the ethics committee (CNREEA C2EA-55), and approved by the Minister of Higher Education, Research and Innovation (Ministère français de l'Enseignement supérieur, de la Recherche et de l'Innovation, approval number: 2015121515281004). All experiments were carried out in accordance with the approved protocol.

\subsubsection{Image acquisition}

To image the bone microstructure and vasculature simultaneously in 3D, we used contrast-agent SR- $\mu \mathrm{CT}$. Imaging experiments were performed at the European Synchrotron Radiation Facility (ESRF), Grenoble, France, on the ID19 beamline. The schematic of the SR- $\mu$ CT imaging setup is shown as Fig. 3. A $2048 \times 2048$ pixel CCD-based detector with effective pixel size of $3.5 \mu \mathrm{m}$ was used to record images at evenly spaced angles of view over a $360^{\circ}$ rotation. The exposure time and X-ray energy were set to $0.15 \mathrm{~s}$ and $26 \mathrm{keV}$, respectively. The acquisition of 2000 radiographs of one sample lasted approximately 8 minutes. After the image acquisition, 3D 32 bits images of $2000 \times 2000 \times 1200$ voxels were reconstructed using a filtered back projection algorithm yielding a cylindrical field of view (FOV) of 7 $\mathrm{mm}$ diameter. The processing involved corrections to take into account beam inhomogeneity, response of the detector and decrease of current during acquisition. Then, the bit depth of reconstructed images were reduced to 8 bits from 32 bits. Finally, the proposed algorithm was applied in the whole SR- $\mu \mathrm{CT}$ images to segment bone and vessels. 


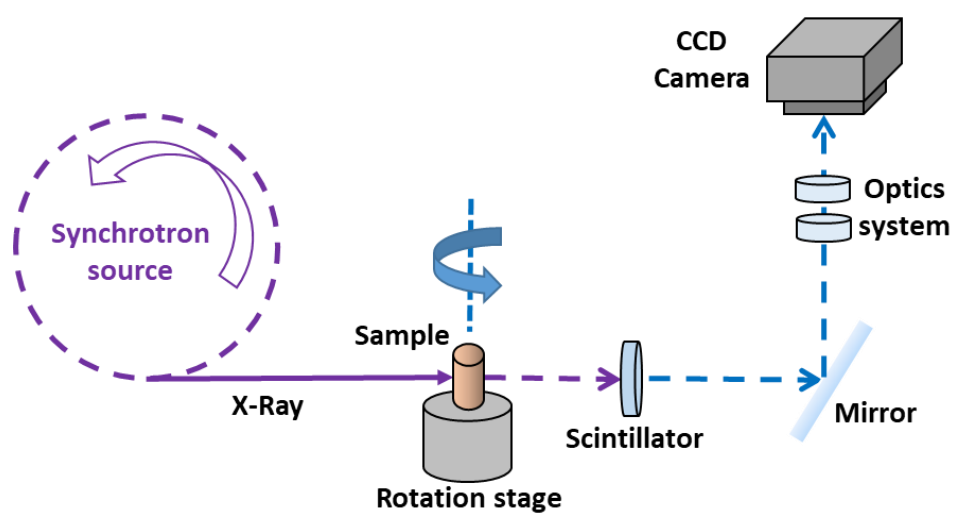

Fig. 3. Schematic of the SR- $\mu \mathrm{CT}$ imaging setup. $26 \mathrm{keV}$ X-rays illuminated a sample mounted on a 3D rotation stage. A CCD-based detector was used for recording images through optics and microscope system.

\subsubsection{Quantitative parameters}

To quantitatively analyze the differences between the healthy group and metastatic group, some anatomical parameters were extracted from the data. The total volume (TV) of the sample was defined as the volume inside the outer contour of the bone. TV, bone volume (BV) and vessel volume (VV) were measured by counting voxels in the corresponding compartments. In order to compare the volume based fractions in the healthy and metastatic group, the normalized ratios BV/TV and VV/TV were calculated. In addition, the local vessel thickness was measured as the diameter of the largest sphere fitting in the vessel at any point of its length (Hildebrand and Rüegsegger, 1997; Langer et al., 2010), and its average VTh was reported.

\subsubsection{Statistical analysis}

Statistical tests were performed on measured BV/TV, VV/TV and VTh, in the healthy and metastatic groups, separately. Lilliefors test was used to verify the normal distributions of parameters in each group. In a case of normal distribution, ANOVA F-test was used to test if there was a significant difference between the two groups. We considered the difference statistically significant at $\mathrm{p}$-value $<0.05$ level.

\section{Results}

In this section, we first examine the proposed method of phase asymmetry based marker-controlled watershed on the $3 \mathrm{D}$ synthetic volumes. Then we apply the proposed method to the real SR- $\mu \mathrm{CT}$ dataset of mouse bone, and extract the quantitative parameters to characterize bone and vessel structures.

\subsection{Assessment of the method on synthetic data}

We first report two examples of the segmentation of Model-1 obtained with various contrast and additive Gaussian noise levels, as shown in Fig. 4, 2D slices from the 3D volumes. Qualitatively, the generated markers and control surface under a relatively low contrast and high noise level were less robust as Fig. 4 (g), leading to a worse watershed segmentation as Fig. 4 (h).

Specifically, to explore the impact of image contrast and the level of noise on segmentation, we generated 189 synthetic volumes for three-class models (Model-1, Model-2 and Model-4) and 70 volumes for a two-class model (Model-3), as explained above. Multi-class structures of each synthetic volume were segmented using the proposed method. In Fig. 5 we show 9 examples of segmentation for each model, obtained under various contrast and noise levels. The panels delineate a representative volume of interest (VOI) for each phantom, obtained at contrast levels of 48, 128 and 208 (or 255) and at noise levels of 10, 30 and 70. Where the algorithm failed to yield a segmentation where the structure was recognizable, which happened only at rather extreme noise levels, we report "no result". Qualitatively, the quality of segmentation decreased with the increase of noise level and the decrease of contrast. 


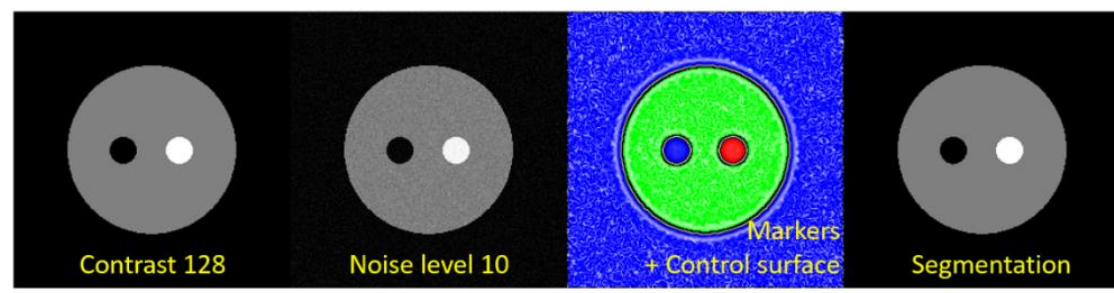

(a)

(b)

(c)

(d)

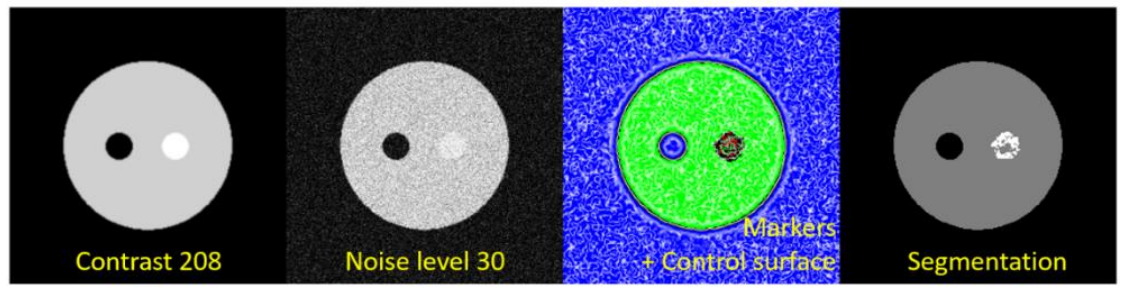

(e)

(f)

(g)

(h)

Fig. 4. Segmentation of synthetic volumes. (a)(e) Noise-free phantoms. (b)(f) Noisy phantoms. (c)(g) Markers overlapping on control surface image. (d)(h) Multi-class segmentation using the proposed method. All images are $2 \mathrm{D}$ slices from the $3 \mathrm{D}$ volumes.

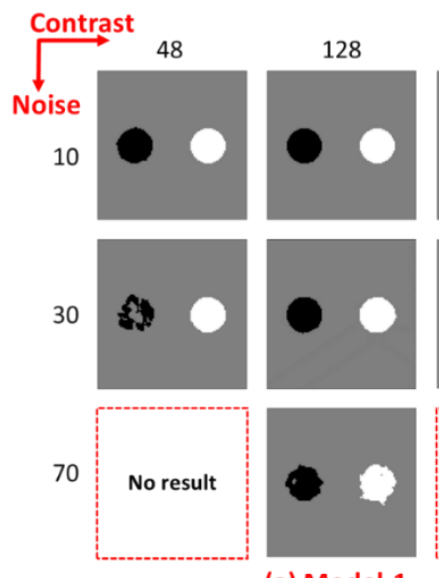

(a) Model-1
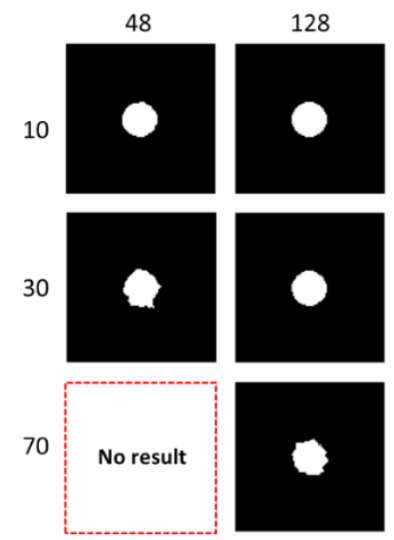

(c) Model-3
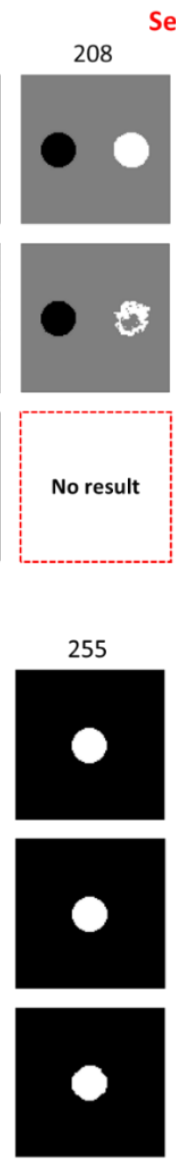

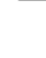

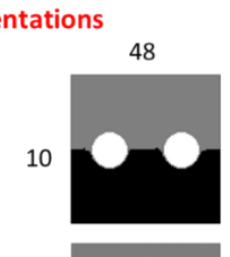
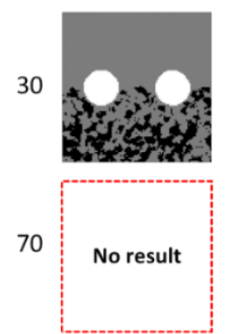

(b) Model-2

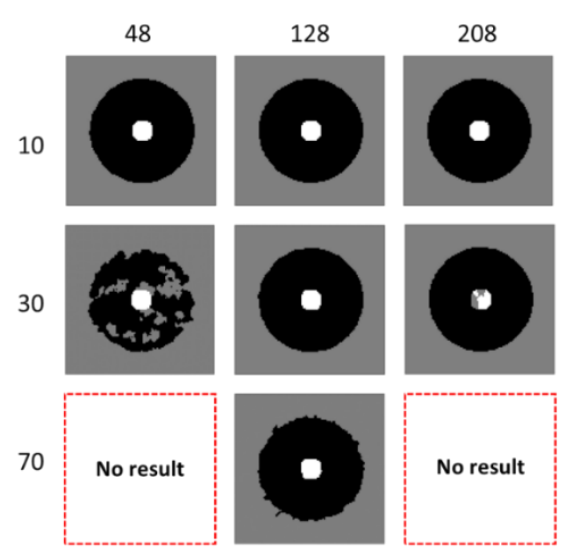

(d) Model-4

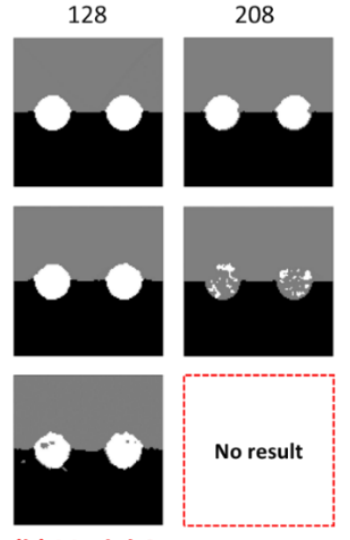

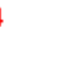

Fig. 5. Impact of image contrast and noise level on segmentation. (a) model-1, (b) model-2, (c) model-3, (d) model4. The 9 examples of segmentation of VOI from each model were obtained using the proposed method. The original synthetic volumes are with contrast of 48,128 and 208 (or 255), as well as noise levels of 10, 30 and 70. All images are $2 \mathrm{D}$ slices from the $3 \mathrm{D}$ volumes. 
Quantitatively, the MCC value for each multi-class segmentation was calculated according to equation (10) as shown in Fig. 6, which illustrates the relationships between MCC and noise levels, separated according to phantom type and contrast. MCC values were plotted over a range of noise levels at each contrast, excluding extremely low values obtained from the failed segmentation as before ("no result", Fig. 5). For example, in Fig. 6 (a), when the noise level is higher than 30, with a bone contrast between 48 and 208, the segmentation algorithm seems to reach its limit. This noise level is extreme compared to the noise in the experimental data, however. In summary, for three-class models of Fig. 6 (a), (b) and (d), in a fixed noise level, the highest MCC values were obtained at contrasts of 128 and 148. This indicates that the proposed protocol works best when the intermediary class has gray levels close to half of the maximum gray level. This can serve as a guideline for optimizing the imaging conditions. For the two-class model of Fig. 6 (c), MCC reaches the highest value at the highest contrast of 255, as expected.

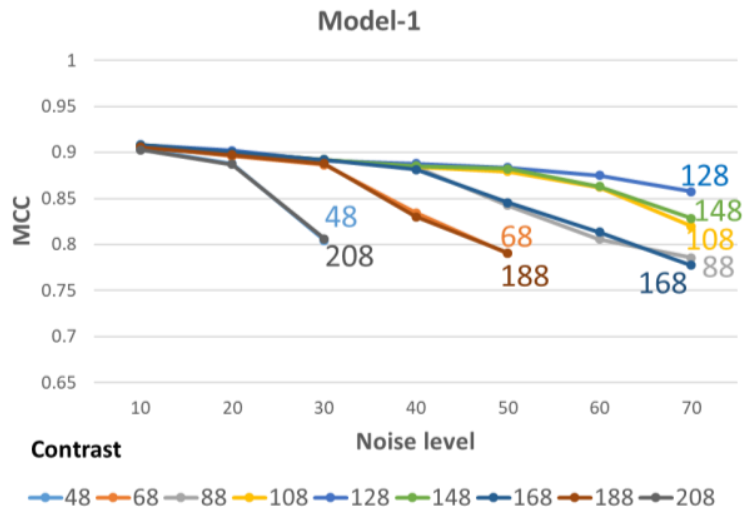

(a)

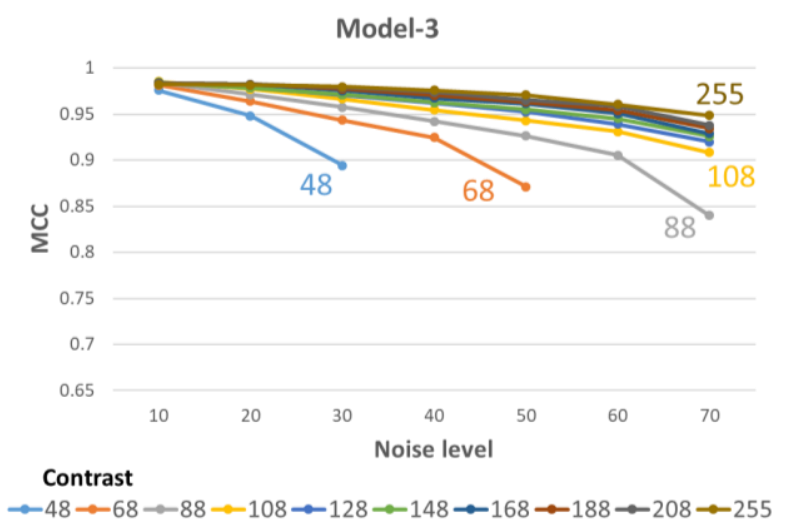

(c)

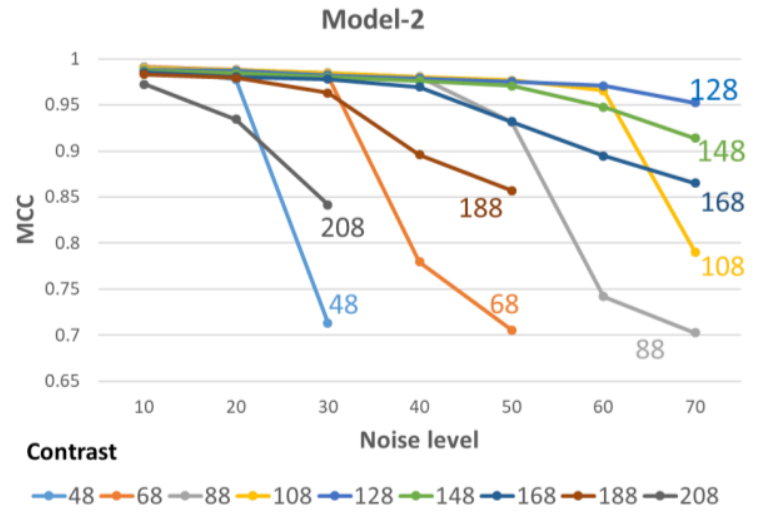

(b)

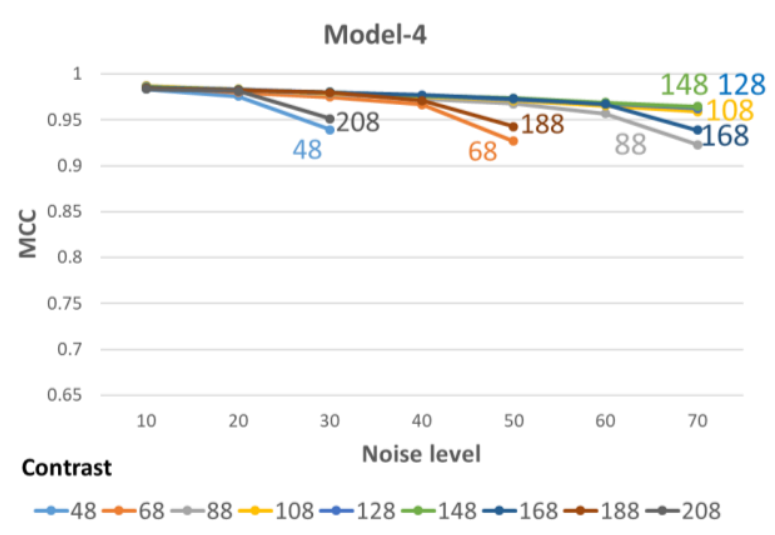

(d)

Fig. 6. Relationship between MCC and noise levels. (a) model-1, (b) model-2, (c) model-3, (d) model-4. Contrast range from 48 to 208 (or 255), and noise levels range from 10 to 70.

Fig. 7 illustrates the capability of thin structure segmentation under a various levels of Gaussian noise. For the purpose of quantifying the thin structure segmentation, we measured 3D local thickness of structure. Specifically, we selected the hourglass structure from the VOI of ground truth as a foreground to generate a new binary volume, and measured its 3D local thickness map. Secondly, the 3D local thickness map was masked with hourglass structure segmentation of Fig. 7 (a-d), and results are shown as Fig. 7 (e-h). As expected, the segmentation quality of thin structure is continuously degraded with increasing noise as the disconnection of structure visible. Lastly, the numbers of voxels at different thicknesses, from the Fig. 7 (e-h), were plotted in the Fig. 7 (i). In a thickness range of 3 to 9 voxels (thin structure), it indicates that thin structure can be well segmented at noise levels of less than 20, using the proposed method. For the segmentation of thick structure (thickness of 10 voxels or more), noise levels have little effects on the number of voxels at each thickness. The thick structures can be well segmented even at the noise level of 60 using the proposed method. 


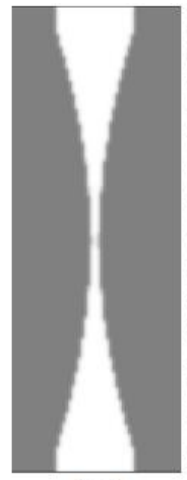

(a)

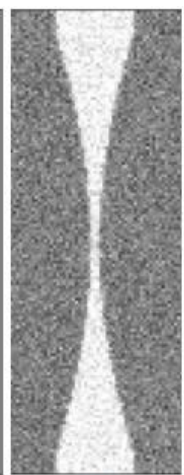

(b)

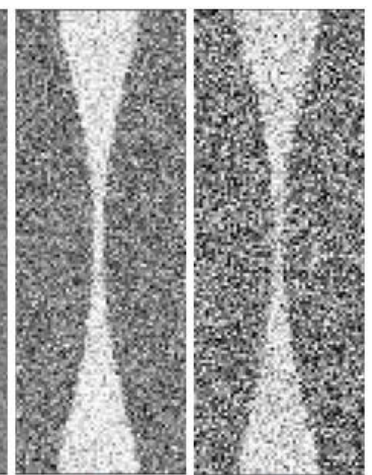

(c) (d)

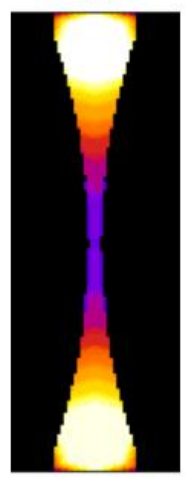

(e)

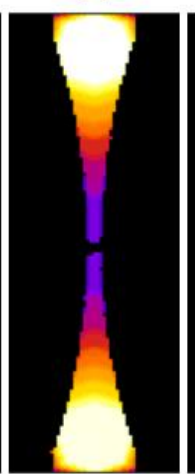

(f)

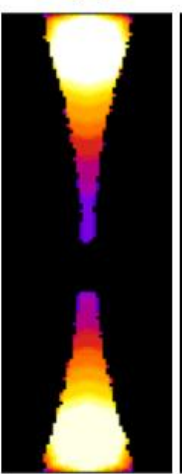

(g)

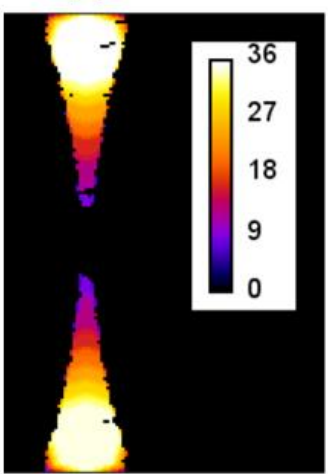

(h)

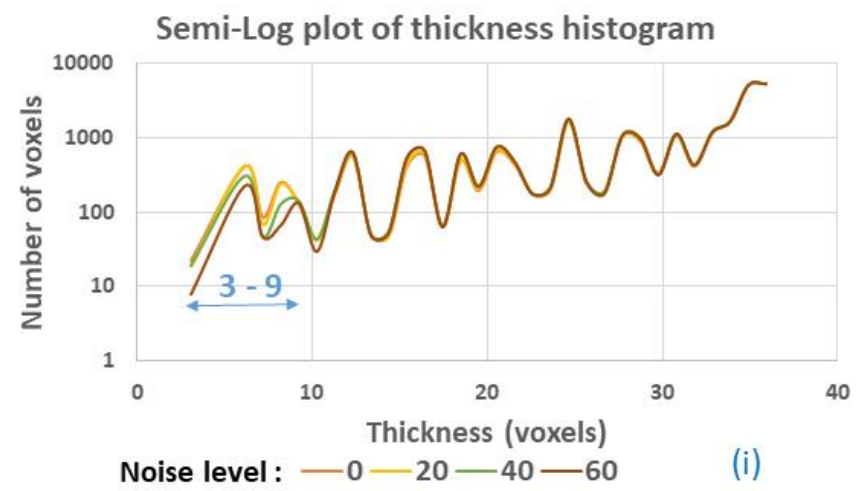

Fig. 7. Performance of thin structure segmentation. (a) Noise-free phantom. (b-d) noisy images with noise levels of 20, 40 and 60, respectively. (e-h) Thickness maps corresponding to segmentation of (a-d). All images of (a-h) are 2D slices of 3D volumes. (i) Thickness histogram based on (e-h).

\subsection{Application to SR- $\mu C T$ mouse data}

\subsubsection{Image processing}

Segmentation results of a representative VOI of an original image are shown in Fig. 8. A zoom on a 2D slice from the 3D original volume is shown as Fig. 8 (a). The red arrow shows the relatively low contrast interface between bone and vessel. The control surfaces were generated using two 3D edge detection methods: conventional image gradient magnitude in Fig. 8 (b) and phase asymmetry in Fig. 8 (c). The gradient magnitude was computed by using a simple finite differences approach with a derivative operator. Conventional gradient was not able to properly resolve the low contrast boundary between bone and vessel indicated by the arrow, which was conversely well detected by the phase 
asymmetry signal. The selection of scales for phase asymmetry depends on image structures. In our case, phase asymmetry with lower scales detects too much edge detail so that useful structures can not be recognized. Conversely, the method with higher scales misses too much detail. As it appears in Fig 8 (c), even though there are some spurious edges detected in homogeneous areas with the phase asymmetry method, they are always covered by a marker in solid areas. Hence they do not influence the marker-controlled watershed segmentation. Qualitatively, the result of segmentation using the phase asymmetry based marker-controlled watershed was much improved compared to hysteresis thresholding or gradient based watershed segmentation. Hysteresis thresholding requires spatial separation between the different compartments. Since this is not the case here, sufficiently low second thresholds cannot be selected to correctly segment the compartments, which leaves a small gap between the structures (Fig. 8(g)). For the watershed algorithm, the conventional gradient did not yield sufficient signal at bone to vessel interfaces to properly separate the two compartments (Fig. 8(b)).
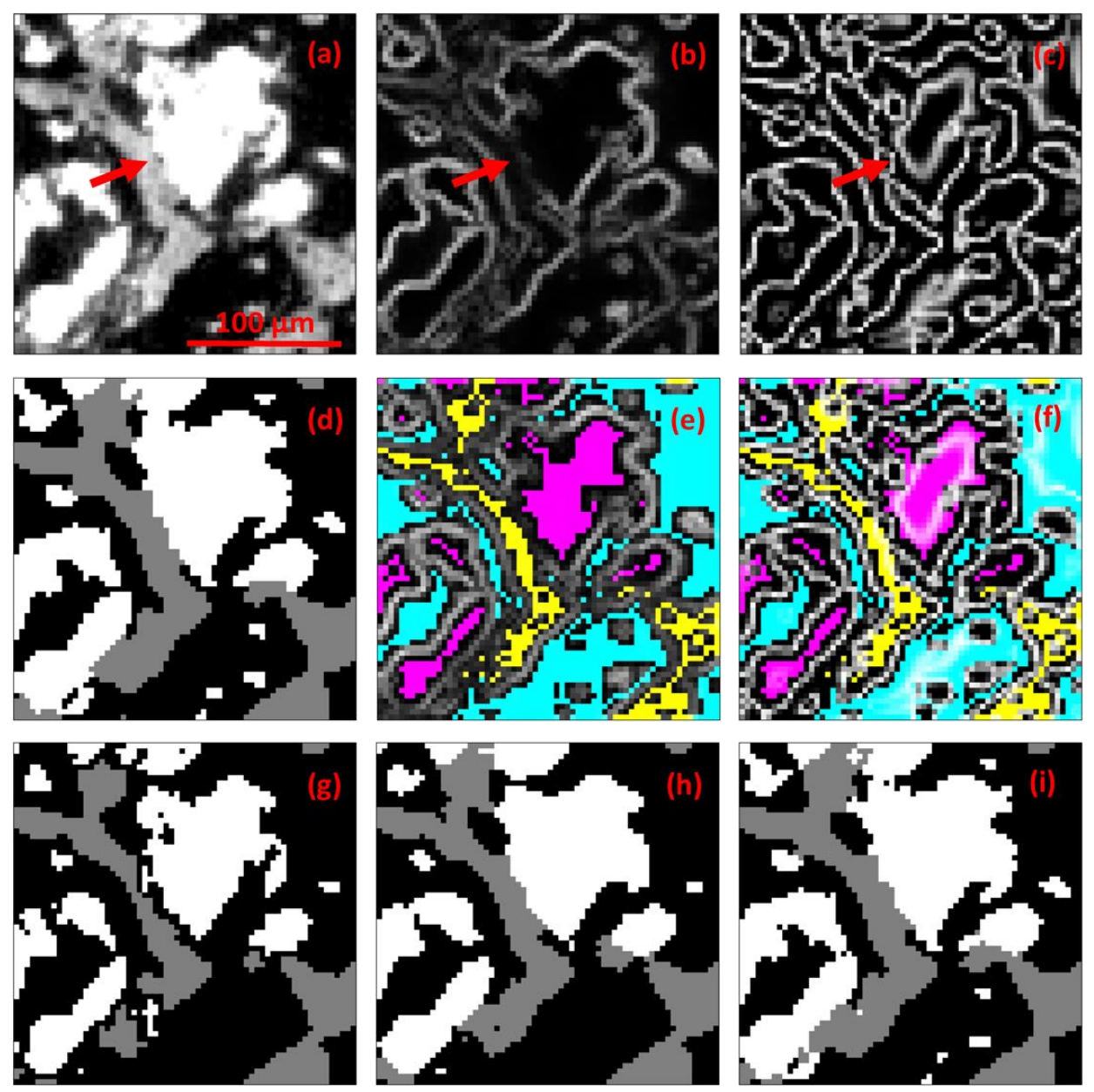

Fig. 8. Representative segmentation examples (VOI) performed with hysteresis thresholds, gradient marker controlled watershed, and phase asymmetry marker controlled watershed (the proposed method). (a) Original image.

(b) Control surface using the image gradient. (c) Control surface using the multiscale phase asymmetry

measurement. (d) Manual segmentation. (e) Vessels marker (red), bone marker (green) and background marker (blue) superimposed on gradient based control surface. (f) Markers superimposed on phase asymmetry based control surface. (g) Hysteresis thresholding segmentation. (h) Marker-controlled watershed segmentation based on gradient (e). (i) Marker-controlled watershed segmentation based on phase asymmetry (f). All images are 2D slices from the $3 \mathrm{D}$ volumes.

\subsubsection{Segmentation quality evaluation}


The segmentation quality was evaluated quantitatively using the Dice coefficient and the MCC by comparing to manual segmentation. A representative volume was manually segmented using VGStudio Max (Volume Graphics $\mathrm{GmbH}$, Heidelberg, Germany). Dice coefficients corresponding to vessel, bone and background compartments were calculated separately, and an overall segmentation quality was also evaluated using multi-class MCC, as shown in the TABLE 1. Especially, there were substantial improvements at the overall segmentation $(M C C=0.94)$ using the proposed phase asymmetry based marker-controlled watershed, comparing to the hysteresis thresholding $(M C C=0.77)$ as well as gradient based marker-controlled watershed $(M C C=0.88)$.

TABLE 1 . Evaluation of segmentation quality

\begin{tabular}{|c|c|c|c|c|}
\hline Evaluation & $\begin{array}{c}\text { Dice } \\
\text { (Vessel) }\end{array}$ & $\begin{array}{c}\text { Dice } \\
\text { (Bone) }\end{array}$ & $\begin{array}{c}\text { Dice } \\
\text { (Background) }\end{array}$ & $\begin{array}{c}\text { MCC } \\
\text { (Overall) }\end{array}$ \\
\hline Hysteresis thresholding & 0.84 & 0.81 & 0.85 & 0.77 \\
\hline Gradient + watershed & 0.92 & 0.91 & 0.93 & 0.88 \\
\hline Phase asymmetry + watershed & 0.97 & 0.92 & 0.97 & 0.94 \\
\hline
\end{tabular}

\subsubsection{Statistical analysis}

To compare the healthy sample with the metastatic sample qualitatively, 3D volume renderings of segmentation using the proposed method are shown as Fig. 9. The metastatic sample in Fig. 9 (d) shows evident large bone lesions, compared to the healthy sample in Fig. 9 (a). In addition, the vessels in the metastatic sample are more numerous and thicker than those in the healthy sample, as evidenced in the comparison between Fig 9 (e) and (b). This may indicate an increased and abnormal vascularization due to the bone metastases, as expected. The plots of BV/TV, VV/TV and VTh in the healthy and the metastatic groups are shown as Fig. 10. Normal distributions of BV/TV, VV/TV and VTh in each group were verified using the Lilliefors test ( $\mathrm{p}$-value $>0.05$ ). ANOVA F-test ( $\mathrm{p}$-value $<0.05$ ) was used to test if there is a significant difference between the healthy and the metastatic groups. BV/TV (median: 0.2673) is significantly lower in the metastatic group compared to in the healthy group (median: 0.3263) as Fig. 10 (a) (ANOVA F-test, p-value $=0.012$, which is consistent with the apparent large metastatic lesions visible in Fig. 9 (d). In addition, there is no significant difference on parameters VV/TV (p-value $=0.715)$ and VTh (p-value $=0.469)$ as Fig. $10(b)(c)$, possibly due to the limited sample size. 


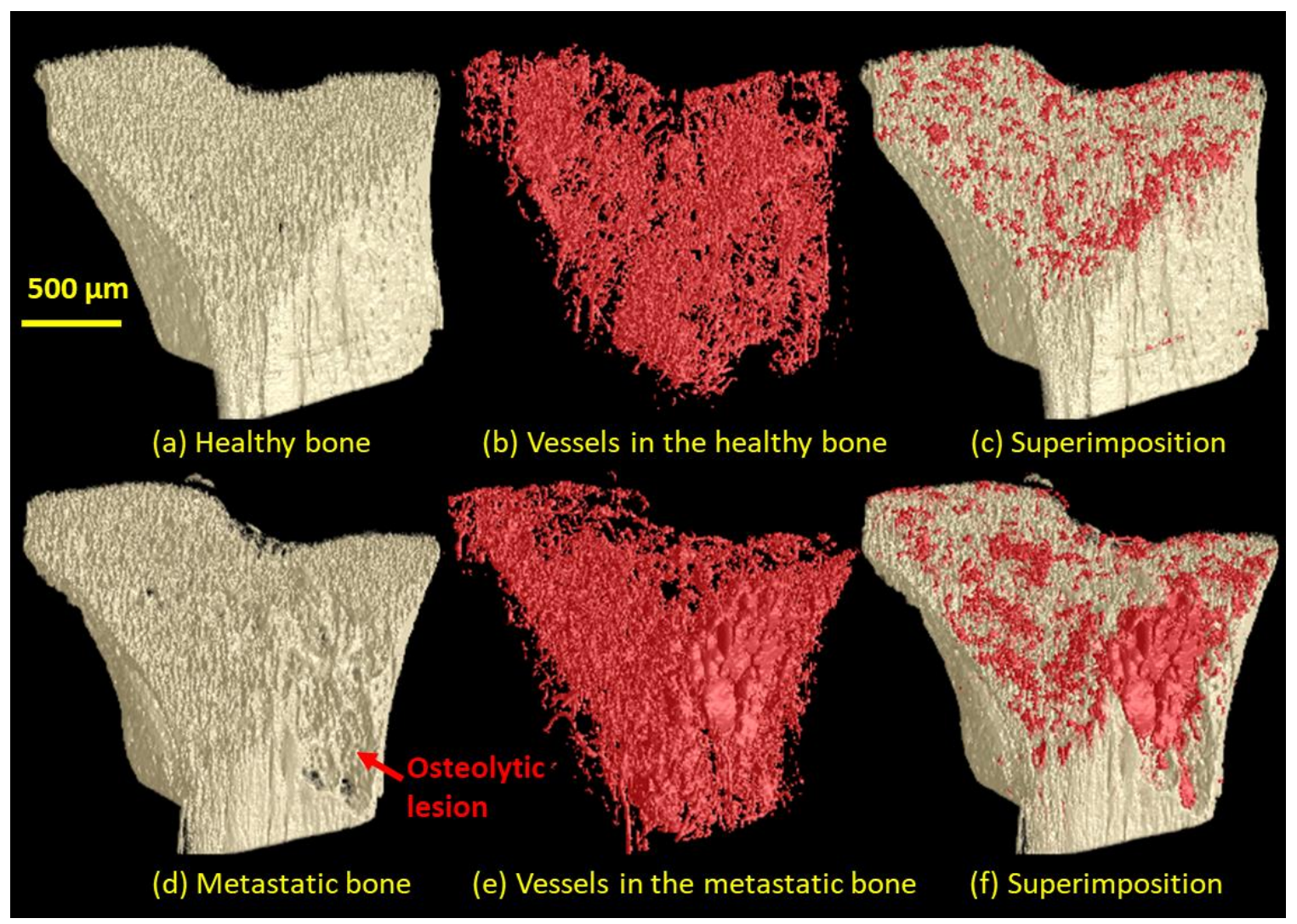

Fig. 9. Qualitative comparison between the healthy and metastatic samples. (a)(b)(c) 3D rendering of bone and vessels in the healthy group. (d)(e)(f) 3D rendering of bone and vessels in the metastatic group.

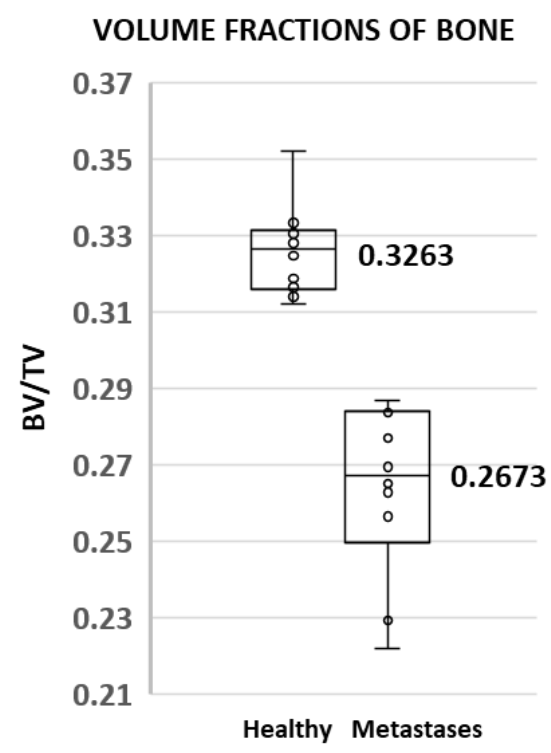

(a)
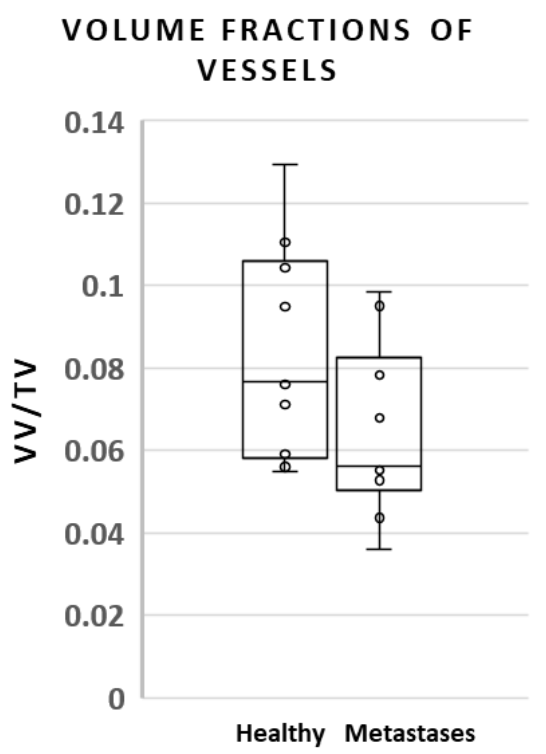

(b)
MEAN THICKNESS

OF VESSELS

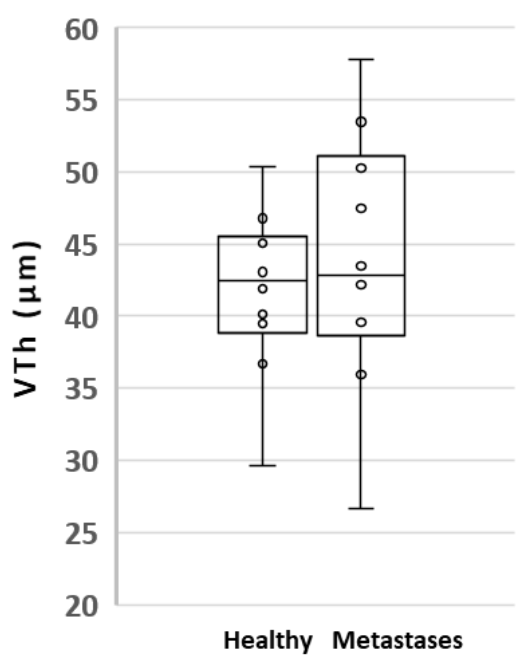

(c)

Fig. 10. Quantitative comparison between the healthy and metastatic samples. (a) Bone volume fraction. (b) Vessel volume fraction. (c) Mean vessel thickness.

\section{Discussion and conclusion}


We presented a method to segment bone and vessels using the marker-controlled watershed and the monogenic signal phase asymmetry. The marker-controlled watershed was used to address the problem that vessels may appear to be in contact with the bone surface in mouse model precluding the correct segmentation of bone and vessels using the previously proposed hysteresis thresholding based protocol. In this algorithm, three classes of markers, i.e. bone, vessels and background were generated using the hysteresis thresholding and morphological filters, minimizing the false positives in the connected components of each class. In addition, we proposed the monogenic signal phase asymmetry as the control surface to improve edge detection at the relatively weakly contrasted bone and vessel interfaces.

To examine the accuracy and robustness of the method, as well as its generalization to other applications, we created 3D synthetic models to simulate a real dataset. Since there may be various contrast ratios between vessels and bone, physically came from the different types of contrast agent, X-ray energy, and range for 8 bits resampling in the real experiment, it is necessary to investigate the impact of image contrast on segmentation quality. In addition, we considered the different noise levels and the segmentation of thin structure. After the multi-class synthetic volumes were segmented using the proposed method, the overall segmentation quality was evaluated using the MCC by comparing to the ground truth. The results indicated that the proposed protocol could work better at the contrast of 128 and 148 in the three-class model, and at the contrast of 255 in the two-class model. In addition, we studied the capability of thin structure segmentation with the proposed method, under various levels of Gaussian noise. The results showed that thin structures (thickness of 3 to 9 voxels) could be well segmented at noise levels of less than 20, and thick structures (thickness of 10 voxels or more) could be well segmented even at the noise level of 60 using the proposed method. In summary, the proposed segmentation method appears to be robust with respect to noise, contrast and thin structure segmentation. Thus, it is a good candidate for a more general application of multi-class segmentation.

We applied the proposed method to the real dataset of mouse bone with breast cancer bone metastases, acquired using SR- $\mu C T$ with contrast agent. The segmentation quality was evaluated using the Dice and the MCC by comparing to manual segmentation. The results showed substantial improvements at each single class segmentation (Dice) and overall segmentation (MCC) using the proposed method, compared to the hysteresis thresholding based method as well as to the gradient based marker-controlled watershed. In addition, for the purpose of quantitative analysis in the healthy and metastatic groups, we extracted three parameters (BV/TV, VV/TV and VTh) to characterize bone and vessels structures. We found that the BV/TV was significantly lower in the metastatic group compared to in the healthy group. Thus, the statistical analysis revealed that bone volume fraction decreased due to the large bone lesions, all in line with previous studies. In addition, there was no significant difference between the healthy and metastatic groups on parameters of VV/TV and VTh, possibly due to the limited sample size. The application to the real dataset demonstrated the utility of the algorithm for the study of bone and vessel microstructures in mouse models. However, in this study, the statistical analysis might be limited by a small sample size (10 samples per group), leading to a low statistical power and less conclusive results. Further biological study can take this factor into account and provide a more statistical basis. The proposed method can be used to gain a better understanding of role of vessels in cancer pathology and can help us better describe angiogenesis involvement in bone metastatic processes.

\section{Acknowledgments}

This work was financed by China Scholarship Council and was performed within the framework of LabEx PRIMES ANR-11-LABX-006 of Université de Lyon and in the context of the OncoStarter project METANGIOBONE. The authors acknowledge Marie-Hèlène Lafage-Proust and Bernard Roche from the SAINBIOSE Lab (INSERM U1059, Université de Lyon), Lamia Bouazza and Philippe Clezardin from the Lyos, Lyon (INSERM UMR 1033, Université de Lyon) for the animal protocol and sample preparation. We thank the ESRF for providing access to beamtime and

support through the Proposal MD858 and Cécile Olivier for data acquisition. We also express our gratitude to Pierre Jean Gouttenoire for assistance in computer programming.

\section{References}

Aaron, C., Snehashis, R., Gherman, A., Reinhold, J.C., Jesson, A., Tal, A., Oskar, M., Heinz, H., Mohsen, G., Bram, P., 2020. Evaluating White Matter Lesion Segmentations with Refined Sørensen-Dice Analysis. Sci. Rep. Nat. Publ. Group 10.

Akhtari, M., Mansuri, J., Newman, K.A., Guise, T.M., Seth, P., 2008. Biology of breast cancer bone metastasis. Cancer Biol. Ther. 7, 3-9. 
Bachelier, R., Confavreux, C.B., Peyruchaud, O., Croset, M., Goehrig, D., van der Pluijm, G., Clézardin, P., 2014. Combination of anti-angiogenic therapies reduces osteolysis and tumor burden in experimental breast cancer bone metastasis. Int. J. Cancer 135, 1319-1329.

Beucher, S., Meyer, F., 1990. Morphological segmentation. Academic Press.

Bhabatosh, C., 2011. Digital image processing and analysis. PHI Learning Pvt. Ltd.

Bousson, V., Peyrin, F., Bergot, C., Hausard, M., Sautet, A., Laredo, J.-D., 2004. Cortical bone in the human femoral neck: threedimensional appearance and porosity using synchrotron radiation. J. Bone Miner. Res. 19, 794-801.

Bray, F., Ferlay, J., Soerjomataram, I., Siegel, R.L., Torre, L.A., Jemal, A., 2018. Global cancer statistics 2018: GLOBOCAN estimates of incidence and mortality worldwide for 36 cancers in 185 countries. CA. Cancer J. Clin. 68, 394-424.

Bridge, C.P., 2017. Introduction to the monogenic signal. ArXiv Prepr. ArXiv170309199.

Catarina, P., Inês, V., Ferreira, A.R., Casimiro, S., André, M., Irina, A., Costa, L., 2017. Bone metastasis risk factors in breast cancer. ecancermedicalscience 11.

Chenouard, N., Unser, M., 2012. 3D steerable wavelets in practice. IEEE Trans. Image Process. 21, 4522-4533.

Cooper, D.M., Turinsky, A.L., Sensen, C.W., Hallgrímsson, B., 2003. Quantitative 3D analysis of the canal network in cortical bone by micro-computed tomography. Anat. Rec. Part B New Anat. Off. Publ. Am. Assoc. Anat. 274, 169-179.

Felsberg, M., Sommer, G., 2001. The monogenic signal. IEEE Trans. Signal Process. 49, 3136-3144.

Fradet, A., Sorel, H., Bouazza, L., Goehrig, D., Dépalle, B., Bellahcene, A., Castronovo, V., Follet, H., Descotes, F., Aubin, J.E., 2011. Dual function of ERR $\alpha$ in breast cancer and bone metastasis formation: implication of VEGF and osteoprotegerin. Cancer Res. 71, 5728-5738.

Gorodkin, J., 2004. Comparing two K-category assignments by a K-category correlation coefficient. Comput. Biol. Chem. 28, 367-374. https://doi.org/10.1016/j.compbiolchem.2004.09.006

Hildebrand, T., Rüegsegger, P., 1997. A new method for the model-independent assessment of thickness in three-dimensional images. J. Microsc. 185, 67-75.

Jurman, G., Furlanello, C., 2010. A unifying view for performance measures in multi-class prediction. ArXiv Prepr. ArXiv10082908.

Kuhn, J.L., Goldstein, S.A., Feldkamp, L.A., Goulet, R.W., Jesion, G., 1990. Evaluation of a microcomputed tomography system to study trabecular bone structure. J. Orthop. Res. 8, 833-842.

Langer, M., Prisby, R., Peter, Z., Guignandon, A., Lafage-Proust, M.-H., Peyrin, F., 2010. Simultaneous 3D imaging of bone and vessel microstructure in a rat model. IEEE Trans. Nucl. Sci. 58, 139-145.

Larrue, A., Rattner, A., Peter, Z.-A., Olivier, C., Laroche, N., Vico, L., Peyrin, F., 2011. Synchrotron radiation micro-CT at the micrometer scale for the analysis of the three-dimensional morphology of microcracks in human trabecular bone. PLoS One 6.

Lote, K., Walløe, A., Bjersand, A., 1986. Bone metastasis prognosis, diagnosis and treatment. Acta Radiol. Oncol. 25, $227-232$.

Matthews, B.W., 1975. Comparison of the predicted and observed secondary structure of T4 phage lysozyme. Biochim. Biophys. Acta BBA-Protein Struct. 405, 442-451.

Moore, D.C., Leblanc, C.W., Müller, R., Crisco III, J.J., Ehrlich, M.G., 2003. Physiologic weight-bearing increases new vessel formation during distraction osteogenesis: A micro-tomographic imaging study. J. Orthop. Res. 21, 489-496.

Núñez, J.A., Goring, A., Hesse, E., Thurner, P.J., Schneider, P., Clarkin, C.E., 2017. Simultaneous visualisation of calcified bone microstructure and intracortical vasculature using synchrotron X-ray phase contrast-enhanced tomography. Sci. Rep. 7, 1-9.

Nutter, F., Holen, I., Brown, H.K., Cross, S.S., Evans, C.A., Walker, M., Coleman, R.E., Westbrook, J.A., Selby, P.J., Brown, J.E., 2014. Different molecular profiles are associated with breast cancer cell homing compared with colonisation of bone: evidence using a novel bone-seeking cell line. Endocr. Relat. Cancer 21, 327-341.

Nyangoga, H., Mercier, P., Libouban, H., Baslé, M.F., Chappard, D., 2011. Three-dimensional characterization of the vascular bed in bone metastasis of the rat by microcomputed tomography (MicroCT). PLoS One 6.

Peyrin, F., Salome, M., Cloetens, P., Laval-Jeantet, A.M., Ritman, E., Rüegsegger, P., 1998. Micro-CT examinations of trabecular bone samples at different resolutions: 14, 7 and 2 micron level. Technol. Health Care 6, 391-401.

Prisby, R., Guignandon, A., Vanden-Bossche, A., Mac-Way, F., Linossier, M.-T., Thomas, M., Laroche, N., Malaval, L., Langer, M., Peter, Z.-A., 2011. Intermittent PTH (1-84) is osteoanabolic but not osteoangiogenic and relocates bone marrow blood vessels closer to bone-forming sites. J. Bone Miner. Res. 26, 2583-2596.

Rajpoot, K., Grau, V., Noble, J.A., 2009. Local-phase based 3D boundary detection using monogenic signal and its application to real-time 3-D echocardiography images, in: 2009 IEEE International Symposium on Biomedical Imaging: From Nano to Macro. IEEE, pp. 783-786.

Roche, B., David, V., Vanden-Bossche, A., Peyrin, F., Malaval, L., Vico, L., Lafage-Proust, M.-H., 2012. Structure and quantification of microvascularisation within mouse long bones: what and how should we measure? Bone 50, 390399.

Salomé, M., Peyrin, F., Cloetens, P., Odet, C., Laval-Jeantet, A.-M., Baruchel, J., Spanne, P., 1999. A synchrotron radiation microtomography system for the analysis of trabecular bone samples. Med. Phys. 26, 2194-2204. 
Schneider, P., Krucker, T., Meyer, E., Ulmann-Schuler, A., Weber, B., Stampanoni, M., Müller, R., 2009. Simultaneous 3D visualization and quantification of murine bone and bone vasculature using micro-computed tomography and vascular replica. Microsc. Res. Tech. 72, 690-701.

Sharma, D., Larriera, A.I., Palacio-Mancheno, P.E., Gatti, V., Fritton, J.C., Bromage, T.G., Cardoso, L., Doty, S.B., Fritton, S.P., 2018. The effects of estrogen deficiency on cortical bone microporosity and mineralization. Bone 110, 1-10.

Tulotta, C., Lefley, D.V., Freeman, K., Gregory, W.M., Hanby, A.M., Heath, P.R., Nutter, F., Wilkinson, J.M., Spicer-Hadlington, A.R., Liu, X., 2019. Endogenous production of IL1B by breast cancer cells drives metastasis and colonization of the bone microenvironment. Clin. Cancer Res. 25, 2769-2782.

Wang, D., Vallotton, P., 2010. Improved marker-controlled watershed segmentation with local boundary priors, in: 201025 th International Conference of Image and Vision Computing New Zealand. IEEE, pp. 1-6.

Zhang, X., Xie, C., Lin, A.S., Ito, H., Awad, H., Lieberman, J.R., Rubery, P.T., Schwarz, E.M., O’Keefe, R.J., Guldberg, R.E., 2005. Periosteal progenitor cell fate in segmental cortical bone graft transplantations: implications for functional tissue engineering. J. Bone Miner. Res. 20, 2124-2137.

Zou, K.H., Warfield, S.K., Bharatha, A., Tempany, C.M., Kaus, M.R., Haker, S.J., Wells III, W.M., Jolesz, F.A., Kikinis, R., 2004. Statistical validation of image segmentation quality based on a spatial overlap index1: scientific reports. Acad. Radiol. 11, 178-189. 